\title{
Detecting planetary transits in the presence of stellar variability
}

\section{Optimal filtering and the use of colour information}

\author{
S. Carpano ${ }^{1, \star}$, S. Aigrain ${ }^{2}$, and F. Favata ${ }^{1}$ \\ 1 Astrophysics Division - Space Science Department of ESA, ESTEC, Postbus 299, 2200 AG Noordwijk, The Netherlands \\ e-mail: carpano@astro.uni-tuebingen; Fabio.Favata@rssd.esa.int \\ 2 Institute of Astronomy, University of Cambridge, Madingley Road, Cambridge CB3 OHA, UK \\ e-mail: suz@ast.cam.ac.uk
}

Received 30 October 2002 / Accepted 9 January 2003

\begin{abstract}
Space-based photometric transit searches, such as the ESA mission Eddington (planned for launch in 2007), are expected to detect large numbers of terrestrial planets outside the solar system, including some Earth-like planets. Combining simplicity of concept with efficiency, the transit method consists in detecting the periodic luminosity drop in stellar light curves caused by the transit of a planet in front of its parent star. In a previous paper (Aigrain \& Favata 2002, hereafter Paper I), we developed a Bayesian transit detection algorithm and evaluated its performance on simulated light curves dominated by photon noise. In this paper, we examine the impact of intrinsic stellar variability. Running the algorithm on light curves with added stellar variability (constructed using data from the VIRGO/PMO6 instrument on board SoHO) demonstrated the need for pre-processing to remove the stellar noise. We have developed an effective variability filter, based on an ad-hoc optimal approach, and run extensive simulations to test the filter and detection algorithm combination for a range of stellar magnitudes and activity levels. These show that activity levels up to solar maximum are not an obstacle to habitable planet detection. We also evaluated the benefits of using colour information in the detection process, and concluded that in the case of Eddington they are outweighed by the implied loss of photometric accuracy.
\end{abstract}

Key words. planetary systems - occultations - stars: microvariability - methods: data analysis

\section{Introduction}

The search for planets outside the solar system has become one of the most active areas of research in astrophysics in recent years. Starting with the discovery by Mayor \& Queloz (1995) of a giant planet in a close orbit around the Sun-like star 51 Peg, the radial velocity method has allowed the detection of almost 100 exo-planets to this day ${ }^{1}$. Butler et al. (1999) discovered the first planetary system around $v$ Andromedae, containing at least three planets, and the longest-running programs are now becoming sensitive to longer period planets similar to the gaseous giants in our own system (Jones et al. 2002).

However, velocity fluctuations at the surface of the star limit the radial velocity method to massive planets (somewhat below the mass of Saturn). One of the alternative methods capable of detecting terrestrial planets is the transit method, based on observing the short $(\approx 10$ hours $)$ periodic $(P \approx$ months to years) dips in stellar light curves caused by a planet passing in

Send offprint requests to: F. Favata,

e-mail: Fabio.Favata@rssd.esa.int

* Now at IAAT, Sand 1, 72076 Tübingen, Germany.

1 See www.obspm.fr/encycl/encycl.html for an up to date listing and a description of the radial velocity method. front of the star's disk. It provides immediate determination of the orbital period and radius of the planet and permits the simultaneous monitoring of many thousands of stars. Combined with radial velocity observations, the true mass of the planet (free from the inclination degeneracy) and all the orbital parameters can be derived.

A large number of ground-based planetary searches are underway, some of which have produced interesting candidate transits (Udalski et al. 2002; Mallen-Ornelas et al. 2003). These will likely lead to a new phase in extra-solar planetary science, bringing the number of known exo-planets from dozens to statistically significant hundreds or thousands. However, the depth of the transit is determined by the ratio of the planetary to the stellar radius $\Delta F / F=\left(R_{\mathrm{p}} / R_{*}\right)^{2}$, and for an Earth-sized planet $\Delta F / F=10^{-4}$. Atmospheric scintillation prevents the detection of so small a signal from the ground. The daily interruptions in the observations also induce significant aliases in the power spectrum of the light curves which seriously hamper the transit detection process. Several space missions are thus under development, in an attempt to reach the terrestrial planet regime. The moderate size Franco-European mission COROT (CNES/ESA), to be launched in 2005, will perform optimally for short period "Hot Earths". The habitable planet regime will 
be probed by two larger missions, Eddington (ESA) and Kepler (NASA), both with 2007 planned launch dates. The simulations presented in this paper were constructed following the design characteristics of the Eddington mission.

The computational challenge of detecting such a short and low amplitude signal in large, noisy datasets has received much attention recently (Defaÿ et al. 2001; Defaÿ et al. 2002; Jenkins et al. 2002; Kovács et al. 2002). In Paper I we presented a Bayesian algorithm which we tested on simulated light curves with white (photon) noise only. However, the most critical noise source is not white, nor is it at all well known: the vast majority of stars are known to be variable to some degree, and the amplitude of these variations can be very large compared to a transit by a terrestrial planet. Intrinsic stellar variability in the range of frequencies relevant for planetary transit searches is due to the evolution and/or rotational modulation of various types of structures at the surface of the star, from active regions and starspots to granulation cells, themselves thought to arise from internal rotation and convection currents coupled with the star's magnetic field. There are few datasets on any given star which permit the study of this variability over the entire range of timescales involved and with the necessary precision - the Sun is in fact the only star with such a dataset, obtained by the VIRGO instrument on board SoHO. It is therefore very difficult to estimate the level and characteristics of the stellar variability noise likely to affect the light curves planetary transit searches will yield. Nonetheless, we have performed tests to evaluate the impact of introducing Sun-like variability in the simulated light curves used in Paper I, and these show that, without filtering before the detection is attempted, the detection performance decreases significantly.

We have therefore developed a filter based on an aspect of transit searches which already guided the detection algorithm's design: the transit shape is known, give or take small variations due to limb darkening and grazing effects. A Fourier domain filter has been constructed which filters out power in passbands where a transit would produce none. This filter enhances the transit's signal to noise ratio, and whitens the remaining noise. We have investigated the performance of the filter coupled with the detection algorithm using the decision theory analysis presented in Paper I, estimating false alarm and missed detection probabilities using simulated light curves with photon noise (for stars with apparent magnitudes ranging from $V=12$ to $V=17$ ) and Sun-like variability. This was done for the total solar irradiance data from the PMO6 instrument and the narrowchannel, 3-colour data from the SPM instrument. For the case of Eddington, we investigated whether splitting the light into different colour bands, or performing all observations in the red part of the spectrum (where stellar variability has smaller amplitudes), would help towards the detection process.

Section 2 describes the inclusion of variability into the simulated light curves, and the initial tests without filtering. The filter itself, and tests performed on individual light curves, are described in Sect. 3, while tests on the performance of the detection algorithm coupled with the filter are reported in Sect. 4. Section 5 contains a discussion of the implications of this work, while concluding remarks are given in Sect. 6.
Table 1. Parameters of the basic star/planet system used to produce the simulated light curves. Note that the stellar magnitude influences only the photon noise level, not the noise-free light curve.

\begin{tabular}{lllc}
\hline \hline Parameter & unit & symbol & value \\
\hline Time step & $\min$ & $t_{\text {unit }}$ & 15 \\
Planet radius & $R_{\oplus}$ & $R_{\mathrm{p}}$ & 2 \\
Star spectral type & & & $\mathrm{K} 5 \mathrm{~V}$ \\
Star radius & $R_{\odot}$ & $R_{*}$ & 0.74 \\
Star mass & $M_{\odot}$ & $M_{*}$ & 0.69 \\
Limb darkening coefficient & & $C_{\mathrm{ld}}$ & 0.484 \\
Star magnitude & mag & $V$ & 13 \\
Orbital period & months & $P$ & 4 \\
Light curve duration & months & $D$ & 4 \\
Transit duration & hours & $d$ & 10 \\
Transit phase & & $\phi$ & random \\
\hline
\end{tabular}

\section{Impact of raw variability on detection performance}

\subsection{Light curve simulation}

As long term photometric data of sufficient precision are not presently available, all data processing algorithms were tested on simulated datasets. The construction of such a data set is a step by step process, the first two steps being identical to the case described in Paper I.

\subsubsection{Noise free light curves}

First, noise free light curves for any required star/planet configuration, with or without transits, were produced using the UTM (Universal Transit Modeler) software by Hans Deeg (Deeg 1999). UTM is capable of modeling all kinds of configurations involving any number of stars, planets, moons, and rings. The current version of UTM only includes circular orbits, and calculates the light curves in white light only. Stellar light reflected by non-luminous objects such as planets is not taken into account. For the present work a single star/single planet configuration was used, with a 1 to 2 Earth radii planet orbiting a $\mathrm{K} 5 \mathrm{~V}$ star with $V=12$ to 17 . A K5V star was chosen as a compromise: brighter, early-type stars have better photometry and are less variable, but smaller, late type stars are more numerous and the transits are deeper. The detailed parameters of the system are shown in Table 1. All the light curves used in this work have the parameters listed in the table unless otherwise stated. The observer was assumed to be in the orbital plane of the system. The phase of the transit events was chosen at random. The light curves have a sampling of $15 \mathrm{~min}$.

\subsubsection{Adding photon noise}

Photon noise was then added, based on the photon count levels expected from the Eddington baseline design for a star of a given magnitude: with a collecting area of $0.6 \mathrm{~m}^{2}$ and a system throughput of $70 \%, \approx 50$ detected photons/s are expected for a 
G2V star ${ }^{2}$ with $V=21.5$ (Favata et al. 2000). As Poisson noise is well approximated by a Gaussian distribution at these count levels, such an approximation was used. The small variation in the count level during a transit was considered negligible, and a constant variance, equal to the mean count level over the entire light curve, was used. Noise was inserted additively:

$$
s_{i}=r_{i}+n_{i}
$$

where $r$ represents the noise-free light curve (later referred to as the "reference" signal, $n$ is the noise and $s$ is the resulting signal. For each point $i$ in the light curve, a noise value $n_{i}$ is drawn from a Gaussian distribution with zero mean and variance $\sigma^{2}=\sqrt{\left(n_{\mathrm{ph}}\right)}\left(n_{\mathrm{ph}}\right.$ being the mean photon count in one sampling time):

$P\left(n_{i}\right)=\frac{1}{\sqrt{2 \pi \sigma^{2}}} \exp \left(-\frac{n_{i}^{2}}{2 \sigma^{2}}\right)$.

\subsubsection{Inserting white light variability}

White light variability was then introduced by multiplying the light curves by (normalised) portions of data from PMO6, rebinned to 15 min sampling. The data spans the 1996 to 2001 period, i.e. it covers the rising half of cycle 23 . Three representative sections were chosen at low, medium and high activity levels respectively, in order to investigate the impact of different activity levels (see Fig. 1). They were chosen to be representative of a particular feature of solar variability patterns, for example containing modulation on the time scale of the solar rotation period (low activity) or signatures of large sunspots (high activity). Care was taken to avoid long data gaps, although there are regular gaps of a few hours, and in the medium and high activity samples, of a few days. This constraint resulted in the sections being only 7 months long, so that they had to be repeated to achieve the full duration of a simulated light curve. The downward trend visible in the raw data, due to instrumental decay, was removed by subtracting a linear fit from the data (only relative irradiance variations were of interest). The photon noise contained in the solar data was ignored, as it is small compared to the added noise.

Examples of simulated light curves including low and high variability, are shown in Fig. 2 . The transits are clearly visible at low activity but at high activity the amplitude of the transit is smaller than the amplitude of the brightness variations, hence transit detection is expected to be difficult.

\subsubsection{Inserting coloured variability}

Besides using the white light PMO6 data, we have studied whether the use of colour information might improve the detection capacity. The VIRGO experiment comprised a narrowchannel, 3-colour instrument, SPM, which measured irradiance variations in $5 \mathrm{~nm}$ bandpasses centered on 402, 500 and $862 \mathrm{~nm}$. Such narrow bandpasses are unrealistic in the case of photon-noise limited transit searches, where the number

\footnotetext{
2 As the simulations were carried out for a $\mathrm{K} 5 \mathrm{~V}$ star, redder than a G2V star, the photon noise is likely to be a slight over estimate.
}
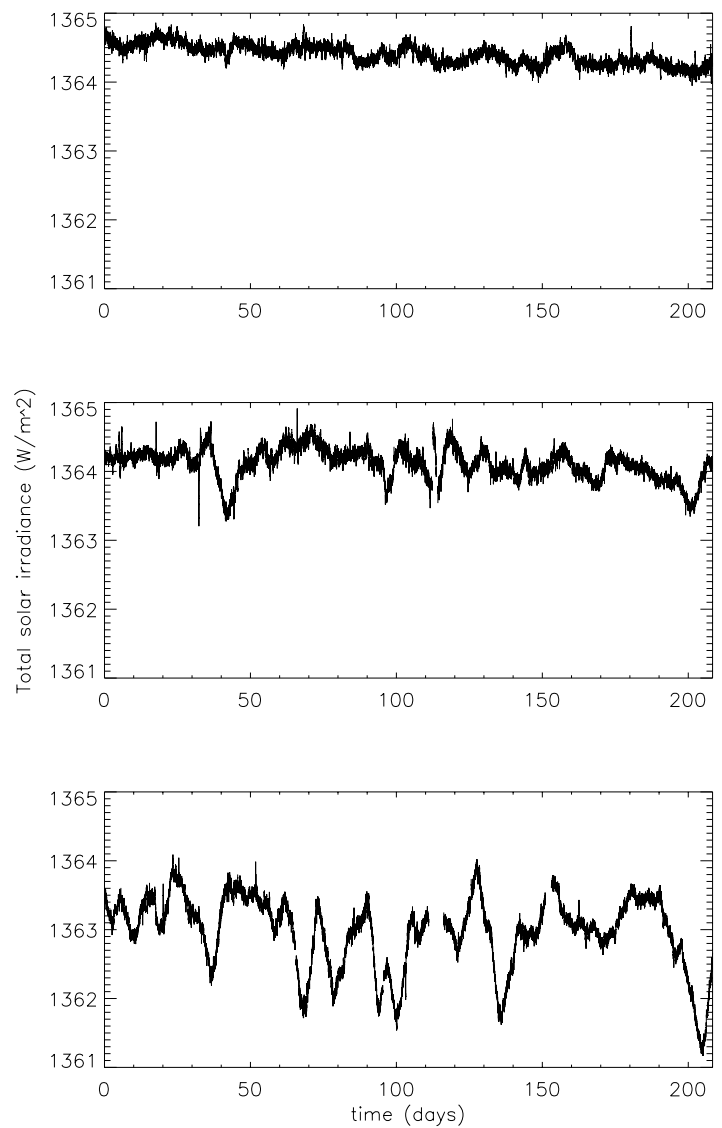

Fig. 1. Total solar irradiance variations $\left(\mathrm{W} / \mathrm{m}^{2}\right)$ from PMO6 data at low (top panel), medium (middle panel) and high (bottom panel) activity.

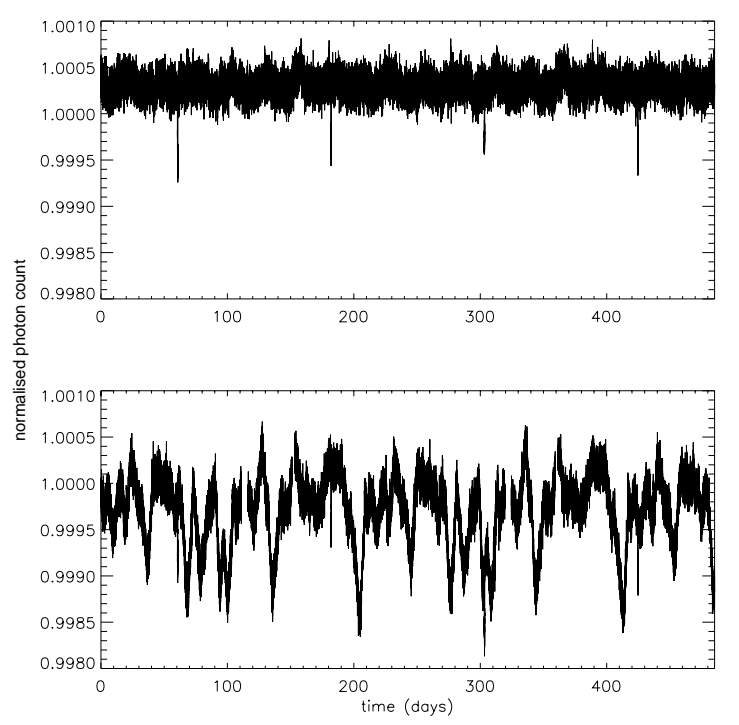

Fig. 2. Simulated light curves in white light containing Sun-like variability from PMO6 corresponding to low (top panel) and high activity (bottom panel).

of detected photons must be maximised. Three "virtual" filters with wide band passes were therefore defined:

- blue: approximate bandpass 390 to $450 \mathrm{~nm}$;

- green: approximate bandpass 450 to $600 \mathrm{~nm}$;

- red: approximate bandpass 600 to $850 \mathrm{~nm}$. 

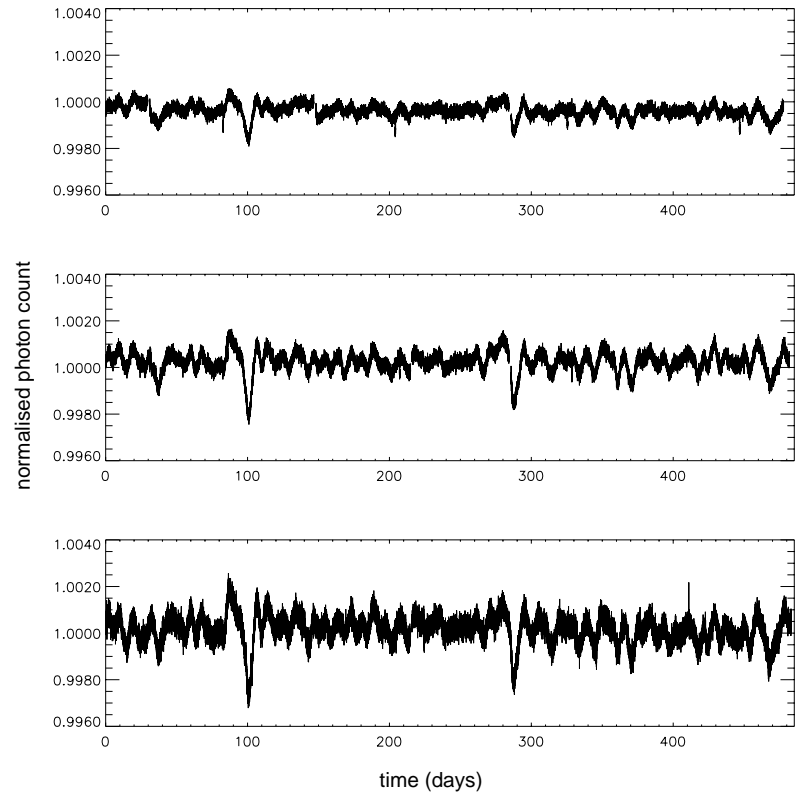

Fig. 3. Simulated light curves containing stellar variability from SPM in red (top panel), green (middle panel) and blue (bottom panel).

The fraction of the light output from a G2V $\operatorname{star}^{3}$ falling into each filter was then computed on the basis of the Eddington baseline design (Favata et al. 2000): 16\% of the photons pass through the blue filter, $44 \%$ through the green filter, and $67 \%$ through the red filter. Note that the total is larger than $100 \%$, as there is marginal overlap between the filters. Coloured light curves were then simulated, assuming that the relative irradiance variations were identical in each filter to those measured in the corresponding SPM channel, but using the filter throughputs listed above to compute the photon noise to add to the data. Figure 3 shows example simulated light curves in red, green and blue (high activity). Both the amplitude of the irradiance variations and the photon noise are less for the red case. Transit detection is thus expected to be easier in the red than in the other two channels. However, the photon noise in the red is higher than in white light, as some photons have been discarded.

\subsection{Tests with raw variability}

A method for evaluating the performance of the transit detection algorithm for a given set of light curve parameters (star, planet, orbit and noise characteristics) was described in Paper I. Large numbers of light curves with identical parameters but different realisations of the photon noise were simulated, with and without transits, and the number of false alarms and missed detections was counted, after the detection threshold was optimised using decision theory. The performance of the algorithm was considered unacceptably low if the mean of the false alarm and missed detection rates exceeded 3\%. In Paper I, where the

\footnotetext{
${ }^{3}$ Using a G2V star to estimate the fraction of the light passing through each filter leads, in the case of a $\mathrm{K} 5 \mathrm{~V}$ star, to a slight underestimate of the photon noise levels in the red and an over-estimate in the blue.
}

white noise only case was considered, the basic star/planet configuration was identical to the system described in Table 1 except that it contained an Earth-size planet and the stellar magnitude was not fixed. The limiting magnitude at which such a system could be detected by a mission with the characteristics of Eddington was determined by going through the evaluation process for a range of stellar magnitudes, keeping the other parameters constant. It was found to be $V=14.5$.

In this paper, in order to assess how the mission performance would be affected by raw stellar variability, the minimum planetary radius that could be detected for a given star and orbital period was determined. Starting again from the basic system described in Table 1, the stellar magnitude was set the white noise limiting case for an Earth-sized planet $(V=14.5)$, and the planetary radius was varied between while 1 and $10 R_{\oplus}$. White light variability at high, medium and high activity was inserted in the light curves as described in Sect. 2.1.3. The minimum planetary radius detected reliably was found to increase to $2 R_{\oplus}$ and low activity, and $3 R_{\oplus}$ at medium and high activity. As the dominating noise source was not the photon noise in this case, the performance is likely to improve only slightly for brighter stars.

Habitable planets have radii between 0.8 and $2.2 R_{\oplus}$. It is therefore crucial for the success of any project aiming to discover habitable planets via the transit method, to reduce the impact of stellar variability, by developing suitable filters.

\section{Variability filtering}

\subsection{The highpass filter}

Inspection of the spectral power distribution of any section of the PMO6 or SPM data immediately reveals that most of the power is concentrated at very low frequencies, below a few $\mu \mathrm{Hz}$ (see Fig. 4, middle panel). A simple highpass filter was therefore the first to be tested. After a number of trial cut-off frequencies were tested, the optimum value was found to be around $2 \mu \mathrm{Hz}$. The detection performance was then evaluated as a function of planetary radius in a manner identical to that described in Sect. 2.2, but applying the highpass filter before running the detection algorithm. The top and bottom panels of Fig. 4 show a light curve with the star/planet system parameters described in Sect. 2.2, with a planet radius of $2 R_{\oplus}$ and high activity, before and after highpass filtering. Although the transits were barely visible before filtering, they are easily seen by eye after. However, the white noise only performance established in Paper I could not be recovered with so simple a filter: compared to the raw variability case, the minimum planet radius to be reliably detected decreased to $2 R_{\oplus}$ for medium and high activity, but even at low activity detection was unreliable for the $R_{\oplus}$ case.

These preliminary tests show that pre-processing of the light curve is a promising direction to follow to curb the variability problem. The failure of the highpass filter for small planetary radii is due to residual power from the stellar variability (due to granulation effects) on timescales of hours. This cannot simply be cut-off, as the transit signal is located in the same 

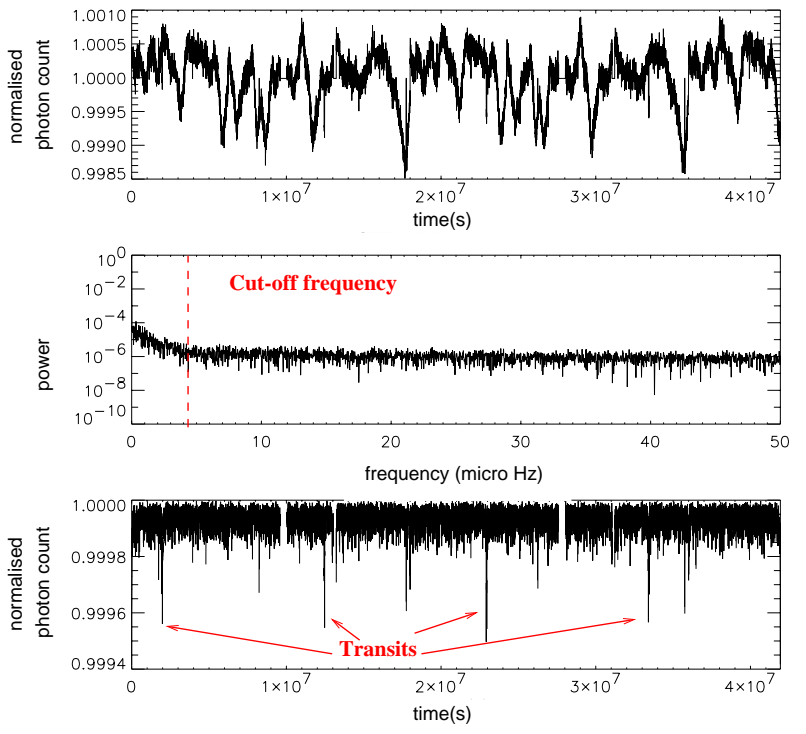

Fig. 4. Simulated light curve in white light, containing variability corresponding to high activity before (top panel) and after (bottom panel) highpass filtering. The middle panel shows the spectral power distribution of the light curve, with the cut-off frequency of the filter overlaid.

frequency range. More sophisticated filters have therefore been investigated.

\subsection{Existing filters}

The COROT and Kepler science teams have investigated two distinct filtering methods, which share the characteristic of being coupled to a matched filter for detection purposes. When using a matched filter for detection, the filter used for the preprocessing stage can be combined directly with the matched filter, so that filtering is applied for each set of model parameters tested.

\subsubsection{COROT: Whitening, matched filter and Bayesian reconstruction}

In the context of the COROT mission, Defaÿ (2001) investigated the use of a Fourier domain whitening filter, which divides the signal by its own spectral power density function, thereby whitening the noise:

$\tilde{h}=\frac{1}{\sqrt{\Phi_{s}(v)+\mu}}$

where:

$\Phi_{s}(v)=S(v) S^{*}(v)$

is the spectral power density of the data, $S(v)$ is its Fourier transform, ${ }^{*}$ denotes a complex conjugate, and $\mu$ is a positive non-zero constant which is necessary to avoid singularities when $\tilde{S}_{s}(v)=0$, and for which they adopted a value of $10^{-6}$. As no a priori knowledge of the noise characteristics are needed, this is known as an adaptive filter. This can be directly coupled with a matched filter. the quantity to be maximised then becomes the correlation function of the data with the combined filter $\tilde{h}$ :

$\tilde{h}=\frac{R(v)}{\sqrt{\tilde{S}_{s}(v)+\mu}}$

where $R(v)$ is the Fourier transform of the reference signal of the matched filter (which depends on the shape and phase of the transit searched for, and if more than one transit is present in the model, on its period). This combined filter is known as an optimal filter. In the case of multi-transits, both the matched filter and another detection method, based on a Bayesian approach combined with the decomposition of both data and reference signals into their Fourier coefficients, were investigated. When the Bayesian method was used, the whitening filter was applied first.

In the mono-transit case the impact of white-light variability, estimated by using a sequence of VIRGO data (corresponding to the activity levels referred to as low and medium in this paper) was found to be significant even using the optimal filter. However, a linear combination of the blue and red SPM channels can be found, which minimises the scatter of the result. Defaÿ (2001) therefore recommended that the observations be performed simultaneously in two broad channels, and the detection performed on a light curve which is a linear combination of the two.

In the multi-transit case, the matched filter was found to perform better than the Bayesian method. However, the Bayesian method can be used post-detection to reconstruct the true (noise-free) transit signal, thereby permitting discrimination of a true planetary transit from, for example, starspot signatures. In that case, the use of colour information was not deemed a priori necessary. However, as the exo-planet runs of COROT are relatively short (5 months, compared to 3 and 4 years respectively for Eddington and Kepler), mono-transit detection is an important aim of the mission. The need for colour information was therefore considered sufficient to justify placing a dispersing element in the optical path of the instrument.

\subsubsection{Kepler: Wavelet domain adaptive filter}

The filter developed by Jenkins (2002) is based on an optimal filter, but takes into account the fact that the characteristics of the noise - i.e. of the stellar variability - are likely to change significantly over the duration of the observations (Kepler will spend its entire operating lifetime of 4 years continuously observing a singe field). A Fourier domain filter cannot account for this, and a wavelet based approach was therefore devised. Dividing the data by its spectral power density, as done by Defaÿ (2001), can be crudely approximated by dividing it by its variance in a number of separate frequency bandpasses. Instead of frequency filters, the Jenkins (2002) filter measures the dependence of noise variance in on both frequency and the time by using wavelet decomposition.

Bootstrap simulations were run to test the performance of the combined wavelet and matched filter on simulated light 
curves including variability from the DIARAD instrument ${ }^{4}$. Jenkins (2002) concluded that solar levels of variability do not prevent the detection of Earth-sized planets around bright $(V=12)$ Sun-like stars by Kepler provided at least 4 transits were present in the light curves, and that smaller planets can be detected if more transits are present. Enhanced activity levels were also simulated by increasing the amplitude of the variability and artificially modifying the sampling times to reproduce the effect of faster rotation rates, and the detection of Earth-sized planets around stars rotation significantly faster than the sun was found to be possible, without the use of colour information.

\subsection{Pre-processing using the optimal filter}

Both matched filters (for example Jenkins et al. 2002) and the Bayesian transit detection algorithms mentioned above (Defaÿ et al. 2001, Paper I) are optimised for Gaussian distributed noise. Photon noise and stellar variability at low activity are roughly Gaussian distributed, but this is not the case for stellar noise at high activity. A pre-whitening filter, as defined in Eq. (3), was therefore applied to flatten the noise spectral power density.

The disadvantage of this filter is that it indiscriminately suppresses power at those frequencies where the raw data shows excess power, whether this power is due to noise or true signal. As a consequence, the signal to noise of the transits in the filtered light curve is reduced when it is converted back to the time domain. This drawback can be avoided if an optimal filter is used. In this case, the noise is whitened while the signal to noise ratio of the transit signal in the time domain is increased. However, we implement the optimal filter as a pre-processing method rather than a detection algorithm (using the Bayesian method presented in Paper I for detection). Equation (5) has therefore been modified to ensure that the filter does not affect the phase of the transits. This is achieved by multiplying the whitening filter by the modulus of the Fourier transform of the reference signal, rather than by its complex value:

$h(v)=\frac{|R(v)|}{\sqrt{\Phi_{s}(v)}+\mu}$.

Note that in the notation adopted here $\mu$ is not enclosed in the square root. As it is a parameter whose sole purpose is to ensure $h(v)$ stays finite at all times, and its value is optimised according to the form of $\Phi_{s}(v)$, this is not important.

Generally, in periodicity searches the form of the reference signal is unknown. In the present case however prior information is available: a simulated transit can be used to calculate $R(v)$. The definition of the reference signal plays a fundamental role in the optimal filter. The phase of the reference transit in the light curve does not influence the modulus of its Fourier transform, and hence can be chosen at random. We will show in Sect. 3.4 that the exact shape (i.e. limb darkening, depth and duration) of the reference transit need not be

\footnotetext{
${ }^{4}$ DIARAD, a part of the VIRGO experiment on board SoHO, is a radiometer which measured variations in total solar irradiance and is almost equivalent to PMO6.
}
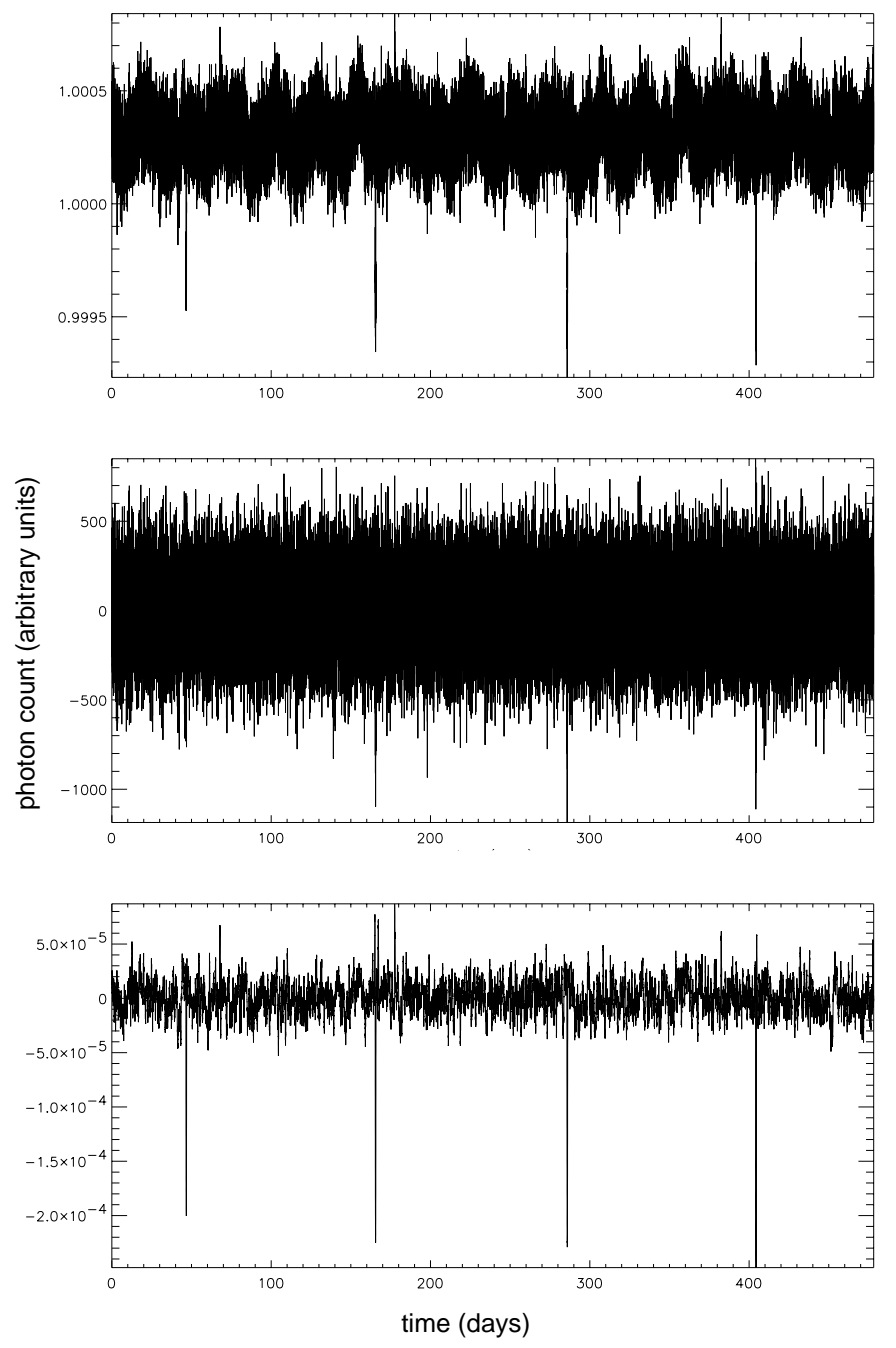

Fig. 5. Simulated light curve in white light, containing variability corresponding to low activity, before filtering (top panel), after whitening (middle panel), and after the optimal filter (bottom panel).

exactly identical to the true signal, provided it is sufficiently close. In this work a reference light curve was simulated using UTM, and was similar to the noise-free light curves described in Table 1, but contained only one transit with random phase.

Figures 5 and 6 show light curves with white light variability before filtering, after the pre-whitening filter and after the optimal filter, at low and high activity respectively. In this case the reference transit exactly matched the true transits. After the pre-whitening filter the noise appears white but the transits are shallower relative to the noise than before the filtering. On the other hand, after the optimal filter, not only is the noise Gaussian, but the signal to noise ratio of the transits is enhanced. The improvement is particularly noticeable at high activity.

\subsection{Influence of the reference planet's radius}

The exact parameters of any transits present in real light curves will not be known a priori. It is therefore important to test the performance of the optimal filter when the reference transits 

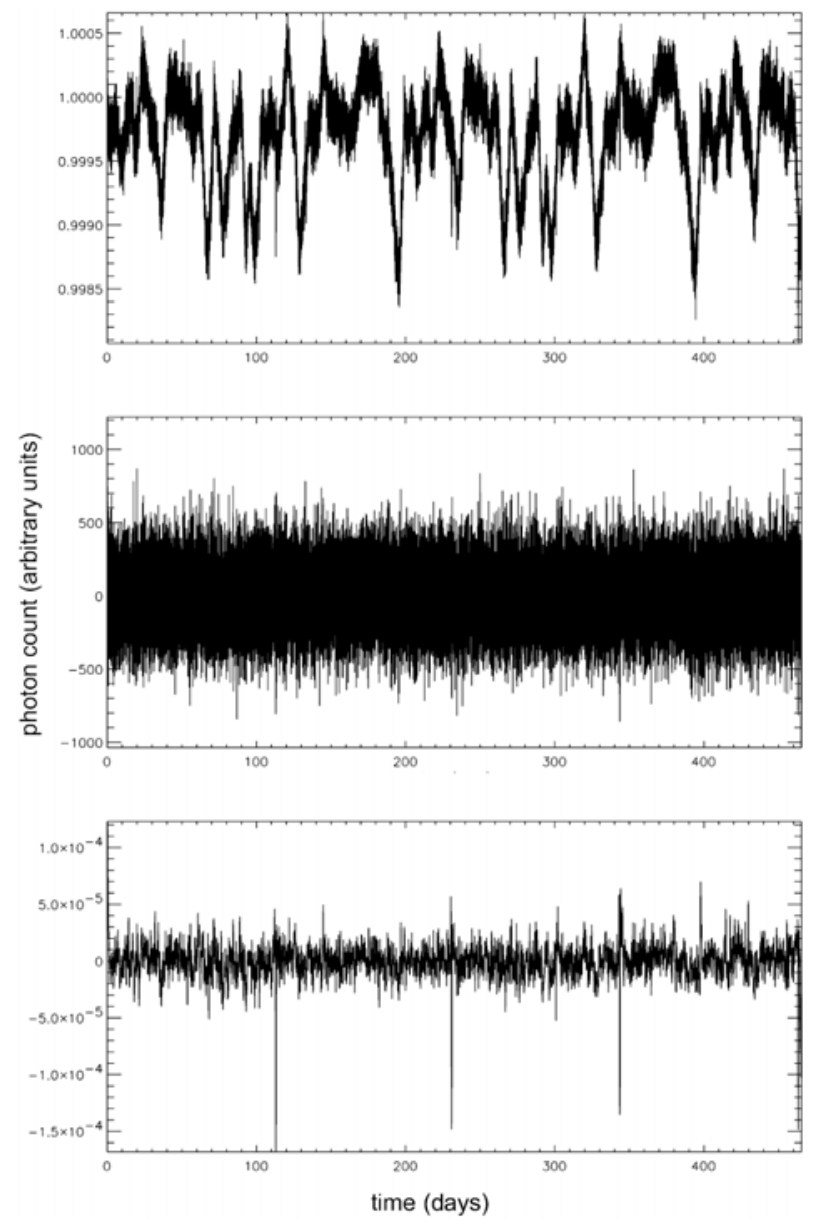

Fig. 6. Simulated light curve in white light, containing variability corresponding to high activity, before filtering (top panel), after whitening (middle panel), and after the optimal filter (bottom panel).

is not identical to the true transit. A priori, we can ignore a number of parameters:

- the period, as the reference signal contains only one transit;

- the phase, as the modulus of the Fourier transform of the reference signal, rather than its complex value, is used in the filter;

- the transit shape, as long as it is similar to the shape produced by ingress and egress and limb-darkening, need not be identical. For example, if a Gaussian was used rather than the transit produced by UTM, the transits in the filtered light curve would appear Gaussian, but the detection performance would not be significantly affected. In any case, the approximate shape of the transit is known a priori, as transits by different planets have similar overall shapes.

An important remark is that, as the shape of the transit is modified by the optimal filter, filtered data cannot be use to measure the parameters of the system (duration, depth, ingress and egress duration, asymmetry of the transits yielding the size, distance, inclination and position angle of the planet.). This filter is useful only for detection purposes. The parameter estimation phase can be performed by fitting a simulated signal to the raw, phase-folded light curve (as can be done with the UFIT
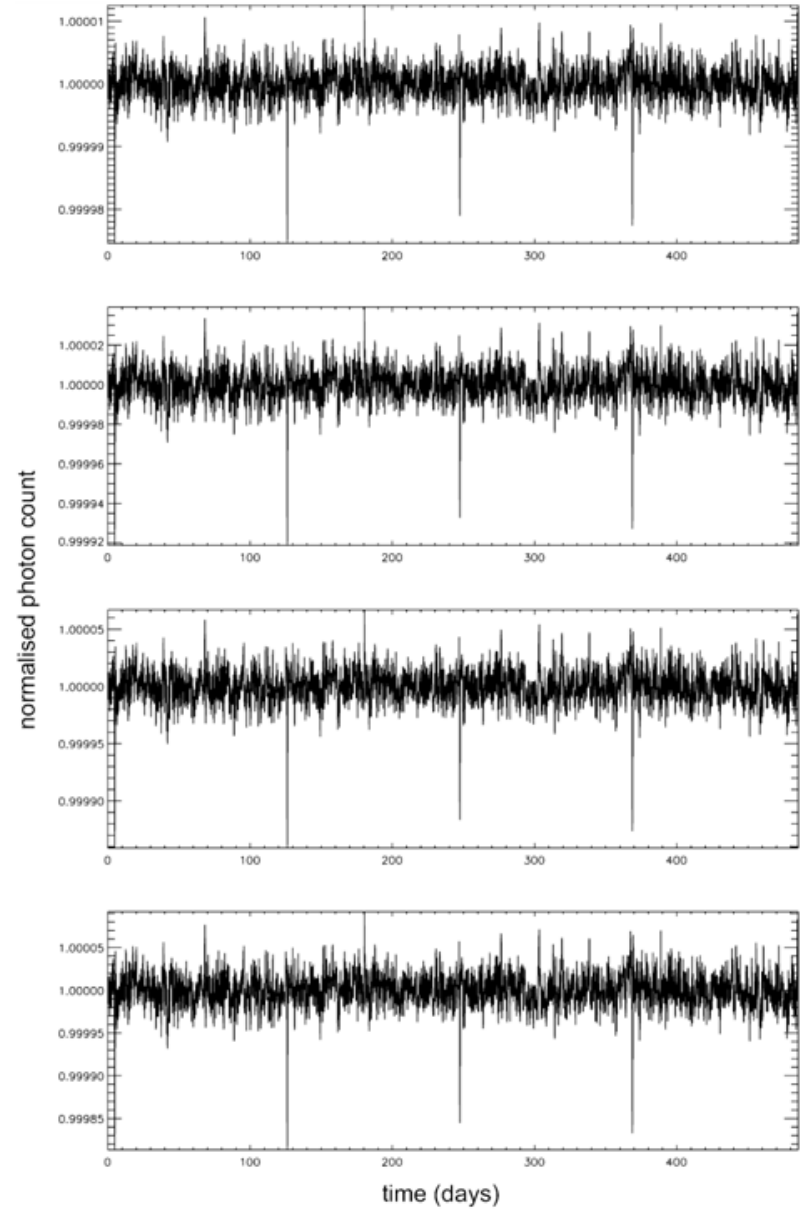

Fig. 7. Output of the optimal filter with reference planetary radii of (from top to bottom) $0.8,1.4,1.8$ and $2.2 R_{\oplus}$ for the input light curve shown in the top panel of Fig. 5 (which contains transits of a $2 R_{\oplus}$ planet).

software package by Deeg 1999). As suggested by Defaÿ et al. (2002), any Bayesian algorithm used for detection purposes can also be used to reconstruct the noise-free transit. This can be done with the algorithm presented in Paper I, but it must be run on the raw light curve once the period and phase are known.

The parameters that might influence the results, and which are not known a priori, are the duration and depth of the transit, determined by the size, period and orbital distance of the planet. To test this, a light curve with the basic parameters listed in Table 1 and low variability, as shown in the top panel of Fig. 5, was fed through optimal filters with reference transits identical in shape and duration to the true transit, but with planetary radii of $0.8,1.4,1.8$ and $2.2 R_{\oplus}$ rather than the true value of $2 R_{\oplus}$. The results are shown in Fig. 7. No discernible difference between the outputs, whether in the shape and depth of the transits after filtering or in the noise. As a result, only a rough estimate of the radius of the planet one is trying to detect is needed. A single reference radius should allow the detection of habitable planets of all sizes. 

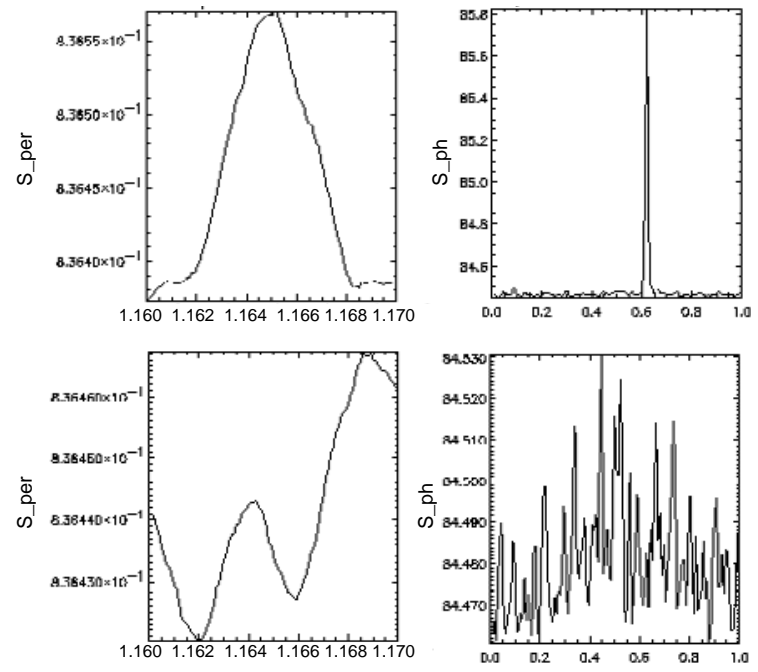

period $\left(15 \times 10^{\wedge} 4 \mathrm{~min}\right)$

Fig. 8. Test statistic distributions for period $P$ and posital phase $p h$ $(p h=\phi / 2 \pi)$ for a light curve with transits (top panel) and a light curve without transits (bottom panel) in the white light, low activity case.

\section{Detection performance after optimal filtering}

\subsection{Method}

The dependence of the detection algorithm's performance on colour and variability was tested by measuring the false alarms and missed detections rates using simulated light curves for a variety of configurations. In the present section we give a brief summary of this method, which was mentioned in Sect. 2.2. This is intended only to present the background of the performance tests, and the interested reader is referred to Paper I for a more detailed description. The description of the detection algorithm itself, which is detailed in Paper I, has not been repeated here. The interval between trial periods was taken to be 15 min rather than 1 hour for increased precision, and trial periods range from 11600 to $11700 t_{\text {unit }}$, i.e. a range of 25 hours around the true period. This is very small compared to the range of periods which would be tested in real data, but the computationally intensive nature of the simulations made the use of a wider period range impractical for the present work. The posital phase $(p h=\phi / 2 \pi)$ was incremented by steps of $d / 2 P$ (where $d$ is the transit duration and $P$ is the orbital period) which is about 0.002 , over the range of periods tested. As was established in Paper I, the algorithm used is not well suited to direct duration determination, so that a single trial duration of $d=10$ hours was used.

For all three activity levels (low, medium and high) and all four colours (white, red, green and blue), 2 sets of 100 simulated light curves were produced (one set containing transits, the other not) with the basic parameters listed in Table 1, for stellar magnitudes from $V=12$ to $V=17$. The algorithm was then run to calculate a detection statistic $S$ for each trial period and phase. Distributions of the period and phase detection statistics $S_{\text {per }}$ and $S_{\text {ph }}$ were computed for each light curve
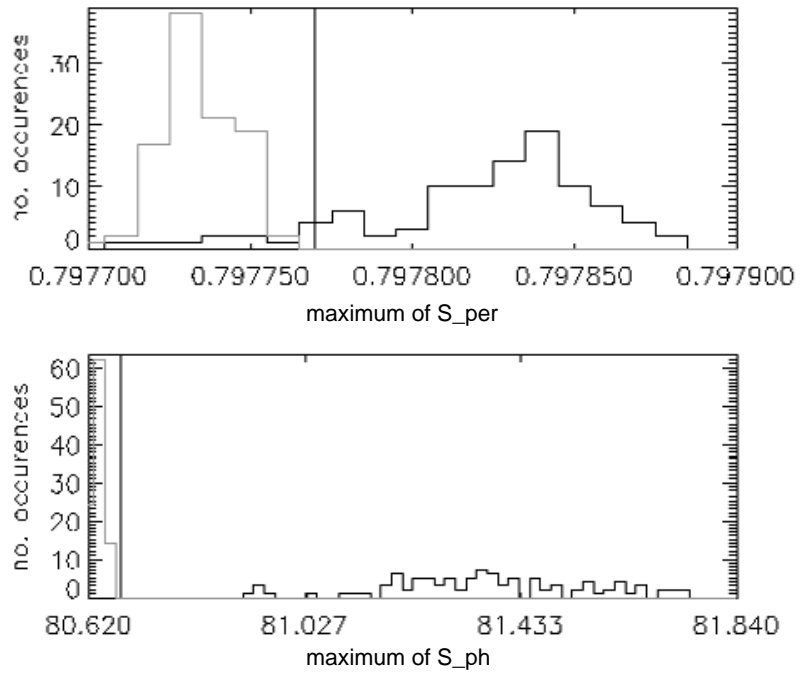

Fig. 9. Histograms of the test statistic distribution maxima for a set of simulated light curves in white light, with variability corresponding to high activity and a stellar magnitude of $V=12$, for the period (top panel) and the phase (bottom panel). Black: histogram corresponding to runs with transits. Grey: histogram corresponding to runs without transits. Vertical solid line: threshold value. The period statistic leads to no false alarms and 8 missed detections over 100 realizations after optimisation of the threshold, the phase statistic to no false alarms and no missed detections.

by marginalising over the phase and the period respectively ${ }^{5}$. Examples of these distributions corresponding to the white light, low activity case, are shown in Fig. 8 for a light curve with transits and one without.

To test the effectiveness of the optimal filter, the test statistic distributions for filtered light curves at low and high stellar activity were compared. An example is shown in Fig. 8 for the low activity case. There are no noticeable differences in the corresponding high activity case, and period and phase are found with small errors in both cases.

It is noticeable that the test statistic distributions are much sharper for the posital phase than for the period. A brief explanation for this goes as follows. If the phase is wrong, even if the period is right, it is likely none of the transits in the data will be matched by the model transits. If the phase is right, whatever the period, at least the first transit will be matched by the model.

Once the test statistic distributions had been computed for the two sets of 100 light curves for a given configuration, the values of the maxima of the distributions were noted in each case. Histograms of these maxima for all 100 noise realisations with and without transits were plotted. An example is shown in Fig. 9 for white light, high activity and a stellar magnitude of $V=12$.

For light curves without transits, the test statistic distributions are noisier and have broader peaks, with lower maxima,

\footnotetext{
5 A combined statistic could be computed by marginalising over phase and period. In the case of two statistics of similar quality but sensitive to different effects, this would improve the performance. In the present case the phase statistic gives significantly better results than the period one, so they have been kept separate.
} 

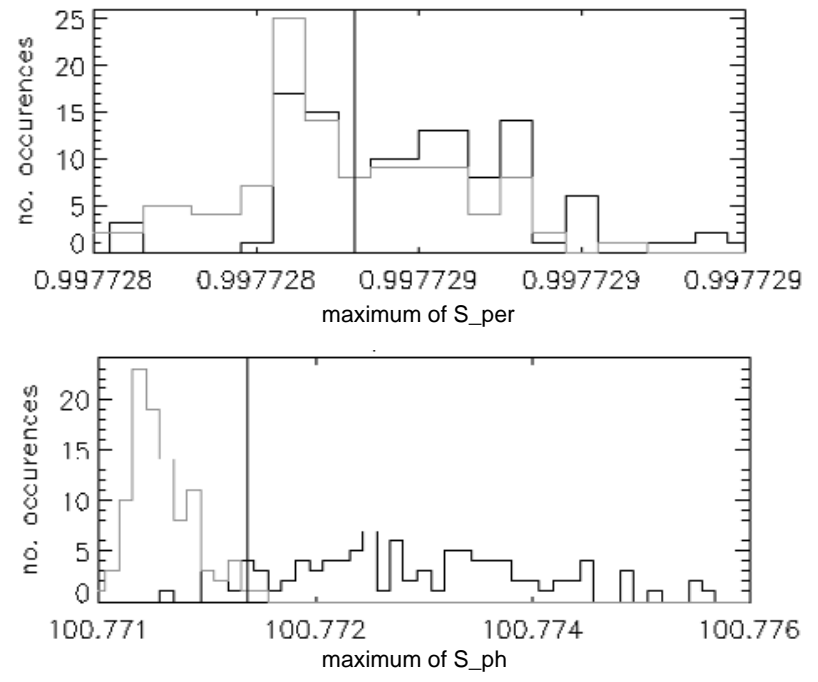

Fig. 10. As Fig. 9 for a stellar magnitude of $V=17$. The period statistic leads to 55 false alarms and 21 missed detections over 100 realizations after optimisation of the threshold, the phase statistic to 2 false alarms and 8 missed detections.

than the corresponding maxima for the light curves with transits. When the algorithm is performing well, as in the case illustrated in Fig. 9, the histograms of the maximas are well separated. A threshold in the test statistic can then be defined, above which any peak in the test statistic is considered significant. This threshold is optimised to minimise the missed detections (i.e. points in the histogram corresponding to the light curves with transits which are below the threshold) and the false alarms (i.e. points in the histogram corresponding to the transit-less light curves which are above the threshold). As the light curves become noisier, so do the test statistic distributions for the light curves with transits, so that the overlap between the two histograms increases. The same method is used to optimise the test statistic threshold, but the resulting number of false alarms and missed detections is higher, as illustrated by Fig. 10.

\subsection{Activity dependence}

The method described above was followed for all three activity levels (in white light) for the full range of stellar magnitudes $(V=12$ to 17$)$, and the false alarm and missed detection rates were measured. The results are shown in Fig. 11. As expected, false alarm and missed detection rates increase with increasing magnitude (as photon noise increases). Importantly however, there are no strong differences between the different activity levels. Variability corresponding to maximum solar activity does not significantly influence the transit detection capacity.

\subsection{Colour dependence}

The procedure was repeated for red, green and blue observations as defined in Sect. 2.1.4. The results are presented in Fig. 12, together with the white light results for comparison purposes. As the results are not activity level dependent, the

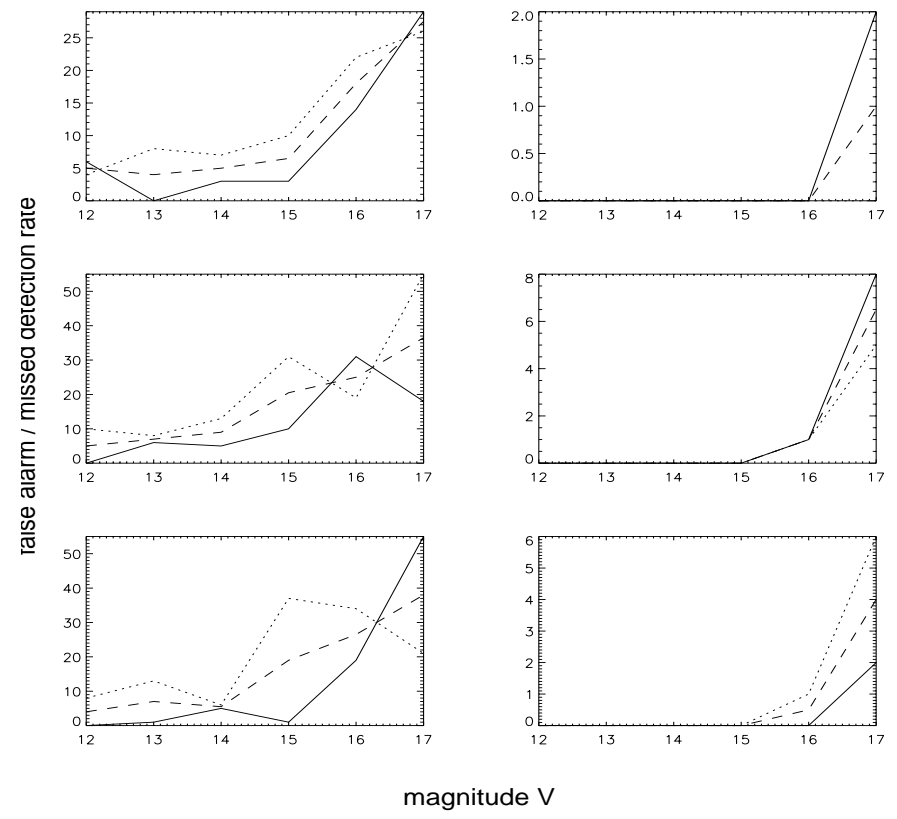

Fig. 11. False alarm (solid lines) and missed detection (dotted lines) rates obtained from white light simulations at low (top row), medium (middle row) and high (bottom row) activity. The left column shows results obtained with the period statistic, the right column with the phase statistic. The dashed lines show the mean of the false alarm and and missed detection rates.

false alarm and missed detection rates shown were averaged over all three activity levels. As expected, the performance improves from blue to green, and from green to red. The period statistic gives slightly better results in the red than in white light, up to as stellar magnitude of $V=15$. For larger magnitudes, photon noise becomes the dominating factor. As the photon count is lower in the red channel than in white light, the performance degrades faster in the red, although the mean error rate is considerable in both cases. The phase statistic however gives better results in white light at all magnitudes, and the mean error rate remains small $(<4 \%)$ up to $V=17$.

\section{Discussion}

Activity-induced microvariability is likely to be the largest intrinsic noise source in the search for transits of habitable planets. Therefore the assessment of its effect on the performance of transit search algorithms is a key element in the design of a space-mission with this scientific goal. The availability of detailed information on microvariability and of high-accuracy, long term light curves for the Sun (and the lack of such information for other stars) make it the best starting point for such investigation. Target stars for transit searches will however span a range of ages and therefore activity levels. To assess for what fraction of target stars the assessment performed here is relevant, the distribution of stellar activity in the solar neighbourhood can be used to characterize how typical the Sun's range of activity levels are. This has been done by Schmitt (1997), who performed a complete volume-limited survey of $\mathrm{X}$-ray emission levels for solar-type stars in the solar neighborhood. This work shows that the activity level of the Sun at 

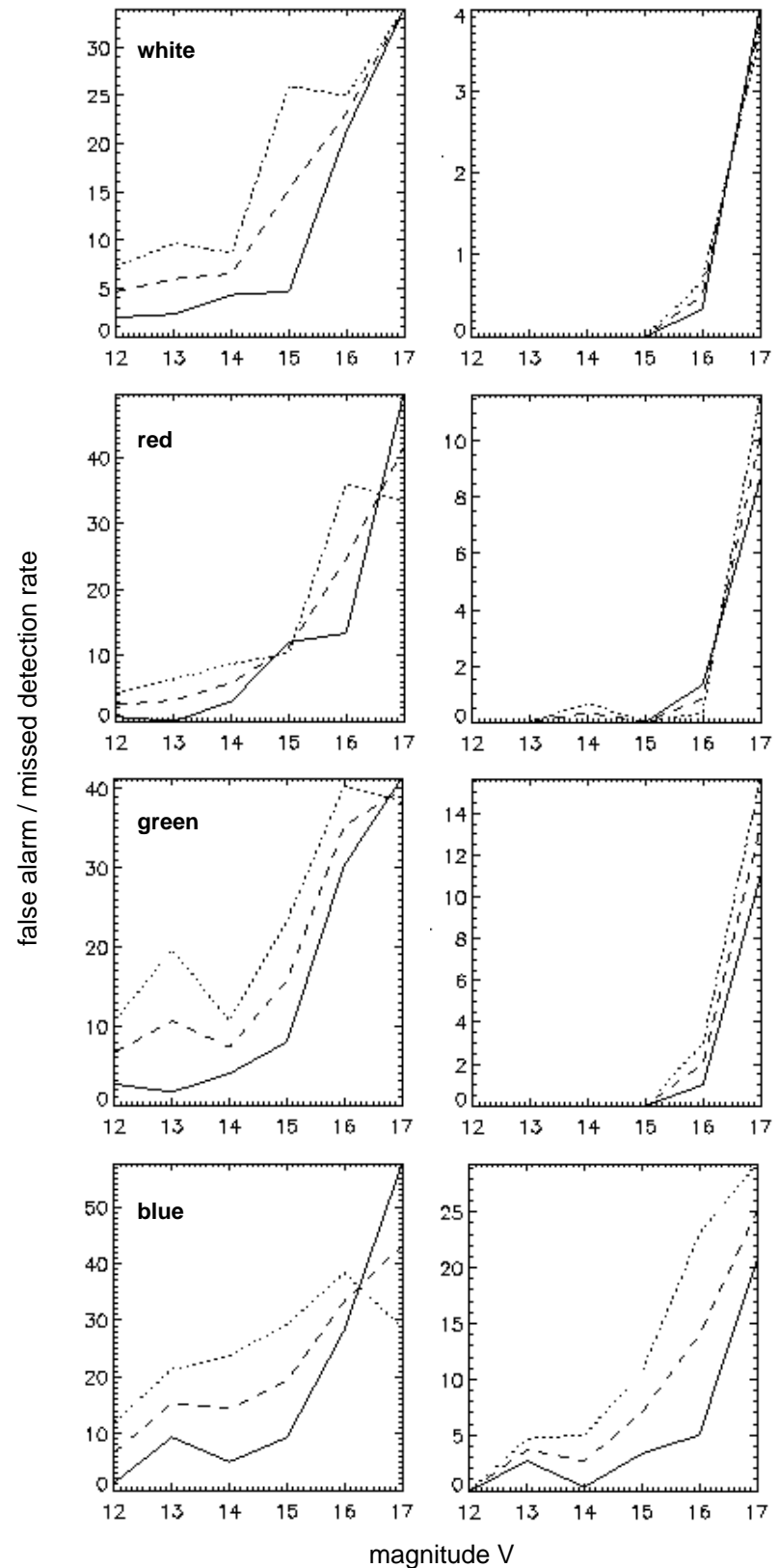

Fig. 12. False alarm (solid lines) and missed detection (dotted lines) rates obtained from white (top row), red (second row), green (third row) and blue (bottom row) light simulations, averaged over all three activity levels. The left column shows results obtained with the period statistic, the right column with the phase statistic. The dashed lines show the mean of the false alarm and missed detection rates.

solar maximum is close to the median average activity level for solar-type stars, so that the results discussed here will be applicable to the "median" star in the target sample of Eddington (or Kepler).

\subsection{Implications for Eddington}

The spectral range to be observed is a fundamental choice for a photometric telescope (whether space- or ground-based), as is whether to observe one single bandpass or more than one. In this respect different space-based missions have made different choices, with e.g. COROT using a prism (allowing to split the light into two or three bands) for its transit search, and Kepler observing in white light.

In the present paper it has been shown that, at the activity levels typical of the median of the target sample, broadband, white light observations are the most effective tool for the search of planetary transits from habitable planets. Coloured light curves do not introduce any improvement in the efficiency of the transit detection process, while unavoidably introducing some additional complexity in the instrumental design. At the same time, the use of a red color produces comparable performance to white light. Thus, a broad-band, white light instrument appears to be the simplest and most effective instrument for transit detection photometry. The Eddington payload aims, in addition to the detection of transits from habitable planets, to perform accurate asteroseismology (for which the advantages and disadvantages of coloured versus white light curves is still not fully assessed), so that the final instrument design will depend from the best compromise among both science goals.

\subsection{Future work}

The results presented here are valid, strictly speaking, for the combination of filtering and detection algorithms used. Work is in progress on an improved (particularly in terms of computational speed) detection algorithm, for which the efficiency of filtering will have to be assessed in detail.

While it has been shown that the detection of transits is not hindered by the presence of stellar activity (up to the solar maximum levels), the filtered light curves are not suitable for the direct determination of the planet's parameters, in particular for the determination of the planet's radius. This has to take place on the unfiltered, original light curve. There, however, the large activity-induced variations make the simple folding of light curves (and parameter fitting on the folded light curve) ineffective. Work is thus also in progress toward the definition of a more robust approach to planetary parameter estimation in the presence of activity-induced noise.

Finally, while as discussed above the results obtained here are valid up to the median activity level of solar type stars, the question is still open on whether the filtering approach described here is also effective for higher activity (and thus microvariability) levels. The key issue there is the lack of detailed knowledge about the characteristics (amplitudes and time scales) of variability for high-activity stars (i.e. the ones with activity levels above median). Starting from a detailed understanding of the solar activity-induced variability and from realistic physical assumptions, an approach to simulate the light curves from high-activity stars is being implemented. Once ready, this will be used to assess up to which activity levels activity-induced noise can be effectively filtered, allowing the performance of transit searches to be assessed across the whole population of solar-type stars.

\section{Conclusions}

Having established that stellar variability is likely to seriously influence the performance of space-based searches for transits 
of terrestrial exo-planets, we have developed an optimal filter to reduce the impact of this variability. This filter is designed to simultaneously whiten the spectrum of the noise and increase the signal to noise ratio of the transits. It has been tested, in conjunction with a Bayesian detection algorithm, on simulated light curves including photon noise levels expected for the Eddington mission and stellar variability corresponding the the range of activity levels exhibited by the Sun.

The detection performance after optimal filtering was estimated by measuring the false alarm and missed detections obtained for large numbers of simulated light curves after optimisation of the detection thresholds. For a $2 R_{\oplus}$ orbiting a $\mathrm{K} 5 \mathrm{~V}$ star and light curves containing 4 transits, tests were made for low, medium and high activity (within the range exhibited by the Sun) in white light and in simulated broad band red, green and blue channels, for apparent stellar magnitudes of $V=12$ to 17 .

The tests have shown that the optimal filter successfully reduces the impact of stellar variability, to the extent that very little difference is observed between the low and high activity results in white light. On the whole better results were obtained in white light than in any of the coloured channels, despite the reduced amplitude of variability in the red channel. This is due to the increased photon noise level in the red channel. Missions such as Eddington and Kepler should therefore be able to detect transits by habitable planets without the use of colour information around all stars with activity levels less than or equal to the Sun's at activity maximum.

Work is underway to simulate realistic light curves of stars more active than the Sun, which will be used to test the detection performance for these stars. An improved version of the algorithm is being tested on existing ground based data from planet searches, together with algorithms designed to detect the large numbers of "classical variables" which form an important secondary science objective of transit finding missions. Besides its higher accuracy, the improved computational efficiency of the new algorithm will allow more extensive simulations to be carried out.

Acknowledgements. S. Carpano was funded by the European Space Agency's Young Graduate Trainee program, and S. Aigrain by studentships from the UK Particle Physics and Astronomy Research Council and the Isaac Newton Trust. The authors wish to thank the VIRGO science team for providing access to PMO6 and SPM data.

\section{References}

Aigrain, S., \& Favata, F. 2002, A\&A, 395, 625

Butler, R. P., Marcy, G. W., Fischer, D. A., et al. 1999, ApJ, 526, 916

Deeg, H. 1999, www.iac.es.galeria/hdeeg/idl_hans_lib/utm

Defaÿ, C., Barge, P., \& Deleuil, M. 2002, A\&A, submitted

Defaÿ, C., Deleuil, M., \& Barge, P. 2001, A\&A, 365, 330

Defaÿ, C. 2001, PhD thesis, Université de droit, d'économie et des sciences d'Aix Marseille

Favata, F., Roxburgh, I., \& Christensen-Dalsgaard, J. 2000, Eddington - A mission to map Stellar Evolution through Oscillations and to find Habitable Planets, Assessment Study Report ESASCI(2000)8, ESA

Jenkins, J. M. 2002, ApJ, 575, 493

Jenkins, J. M., Caldwell, D. A., \& Borucki, W. J. 2002, ApJ, 564, 495

Jones, H. R. A., Butler, R. P., Tinney, C. G., et al. 2002, MNRAS, submitted

Kovács, G., Zucker, S., \& Mazeh, T. 2002, A\&A, 391, 369

Mallen-Ornelas, G., Seager, S., Yee, H. K. C., et al. 2003, ApJ, 582, 1123

Mayor, M., \& Queloz, D. 1995, Nature, 378, 355

Schmitt, J. H. M. M. 1997, A\&A, 318, 215

Udalski, A., Paczynski, B., Zebrun, K., et al. 2002, Acta Astron., 52, 1 